\title{
Discriminative validity of the timed up and go test for community ambulation in persons with chronic stroke
}

\author{
Seung Heon $\mathrm{An}^{\mathrm{a}, \dagger}$, Dae-Sung Park ${ }^{\mathrm{b}, \dagger}$, Ji Young Lim ${ }^{\mathrm{b}}$ \\ ${ }^{a}$ Department of Physical Therapy, National Rehabilitation Hospital, Seoul, Republic of Korea \\ ${ }^{b}$ Department of Physical Therapy, Konyang University, Daejeon, Republic of Korea
}

Objective: The timed up and go (TUG) test is method used to determine the functional mobility of persons with stroke. Its reliability, validity, reaction rate, fall prediction, and psychological characteristics concerning ambulation ability have been validated. However, the relationship between TUG performance and community ambulation ability is unclear. The purpose of this study was to investigate whether the TUG performance time could indicate community ambulation levels (CAL) differentially in persons with chronic stroke.

Design: Cross-sectional study.

Methods: Eighty-seven stroke patients had participated in this study. Based on the self-reporting survey results on the difficulties experienced when walking outdoors, the subjects were divided into the independent community ambulation (ICA) group ( $\mathrm{n}=35$ ) and the dependent community ambulation group $(n=52)$. Based on the area under the curve (AUC), the discrimination validity of the TUG performance time was calculated for classifying CAL. The Binomial Logistic Regression Model was utilized to produce the likelihood ratio of selected TUG cut-off values for the distinguishing of community ambulation ability.

Results: The selected TUG cut-off values and the area under the curve were $<14.87$ seconds (AUC $=0.871,95 \%$ confidence inter$\mathrm{val}=0.797-0.945)$, representing a mid-level accuracy. Concerning the likelihood ratio of the selected TUG cut-off value, it was found that the group with TUG performance times shorter than 14.87 seconds showed a 2.889 times higher probability of ICA than those with a TUG score of 14.87 seconds or longer $(p<0.05)$.

Conclusions: The TUG can be viewed as an assessment tool that is capable of classifying CAL.

Key Words: Community participation, Stroke, Walking, Walking speed

\section{Introduction}

The timed up and go (TUG) test developed by Podsiadlo and Richardson [1] is a simple method used to evaluate functional mobility. The TUG test records the time it takes for a participant to complete the consecutive test of standing up from a chair, walking 3 meters forward, turning $180^{\circ}$, and sitting back down on the chair in order to assess the one's dynamic balance and ambulation ability [2]. It is applicable in the clinical field without special education, and does not re- quire any special equipment or setting. The test results are easy to interpret. The clinicians can observe how patients adjust their position during the TUG test. Therefore, it is useful for understanding functional mobility and ambulation ability [1].

In persons with chronic stroke, the TUG test has been proven to have a high inter-rater reliability (intra-class correlation coefficient $[\mathrm{ICC}]=0.95-0.96)$ and test-retest reliability $(\mathrm{ICC}=0.99)[1,3]$. It has been reported that the TUG test has significant relationships with the affected-side ankle joint

Received: 18 September, 2017 Revised: 13 December, 2017 Accepted: 14 December, 2017

Corresponding author: Ji Young Lim

Department of Physical Therapy, Konyang University, 158 Gwanjeodong-ro, Seo-gu, Daejeon 35365, Republic of Korea Tel: 82-42-600-6419 Fax: 82-42-600-6565 E-mail: deartojay@gmail.com

${ }^{\dagger}$ These two authors contributed equally to this work as co-first author

(c) This is an Open-Access article distributed under the terms of the Creative Commons Attribution Non-Commercial License (http://creativecommons.org/licenses/ by-nc/4.0) which permits unrestricted non-commercial use, distribution, and reproduction in any medium, provided the original work is properly cited.

Copyright $\odot 2017$ Korean Academy of Physical Therapy Rehabilitation Science 
dorsiflexion muscular strength $(\mathrm{r}=-0.67)$, ambulation velocity $(\mathrm{r}=-0.55)$, Berg Balance Scale $(\mathrm{r}=-0.72)$, Bathel in$\operatorname{dex}(\mathrm{r}=-0.51)$, and Functional Gait Assessment $(\mathrm{r}=-0.84)$ [4]. Moreover, the TUG test has the capacity to detect movement change according to the disease period (1 week-3 months) of persons with stroke [5]. The time taken to do the TUG was considered a susceptible criterion of assessment to detect clinical changes ( $9 \%$ change in TUG performance time) [6]. Presently, the TUG test is utilized to evaluate the functional mobility of persons with chronic stroke [7,8]. The previous studies have been performed on fall prediction $[9,10]$ and reaction rate on ambulation ability that may have changed throughout the disease period in the elderly population and in persons with stroke $[5,6]$. The TUG test is known to have $87 \%$ susceptibility [10], which indicates the accuracy of test for fall prediction, and $100 \%$ singularity [11].

Stroke survivors with a neurological disability generally experience difficulties performing movements in consecutive order, such as standing up, walking, and turning from a seated position $[12,13]$. The most essential goal of stroke rehabilitation is to promote their independence in daily activities and participation in community activities [12]. Recently, the mobility and social activities of persons with stroke are being integrated to in order to assist in transitioning into community ambulation [14]. Community walking ability is attributed to physical and psychological factors and extend to external factors such as the individual's social role and life style [15]. In a previous study, community mobility was defined as 8 environmental dimensions: ambient conditions, terrain characteristics, external physical load, attentional demands, postural transition, traffic level, time constraints, and walking distance [16]. Subsequently, the ambulation ability in persons with stroke was compared with the ambulation ability of the people of the community without disability [17]. The differences in people who had disability and no-disability were found in 4 of these dimensions: temporal factors, postural transitions, physical load, and terrain.

The TUG test demands the combination of cognition and coordinated upper/lower-limb movements during testing [18]. Subjects are concentrating on consecutive tasks in order to balance while performing sit-to stand, turning around and returning to the starting point [18]. To do this, they need to have the neural muscular skeletal components of strength, agility, flexibility, and balance $[12,18,19]$. There is complex relationship between the attentional demands to control motor performance and the performance in daily living. Since multidimensional concepts have an effect on the relationship, clinical practitioners are required to analyse factors determining the functional movements necessary in order for stroke survivors to be able to join the community. The relationship between TUG scores and community ambulation ability needs to be taken into account. The purpose of this study was to investigate whether the TUG performance time could indicate community ambulation levels (CAL) differentially in persons with chronic stroke.

\section{Methods}

\section{Participants}

This study examined 120 chronic stroke survivors who had been diagnosed with hemiparalysis and received rehabilitation at hospital. The inclusion criteria were: at least six months after the most recent onset, a cognitive function score $\geq 24$ points per the Mini-Mental Status ExaminationKorea, and gait ability to perform $10 \mathrm{~m}$ or more and TUG test regardless of using an aid such as a cane. They had no medical history such as cardiac, pulmonary, or orthopaedic disorders in the lower extremity. The subjects provided their consent to participate in this study. All of the study procedures were approved by the Institutional Review Boards of Konyang University (IRB No. 2017-066).

\section{Assessment procedures}

This was a cross-sectional study. The general characteristics of participants were collected through charts and interviews, such as age, disease period, diagnosis, and paralyzed area. A physical therapist who had a rehabilitation experience of more than 10 years had conducted the outcome measures CAL and TUG performance. The subjects were categorized according to the CAL, in which they responded. The TUG was performed 3 times for each participant and the data was averaged per group. All assessments were performed within 1 hour, and the participants were provided a resting period (1-minute) between tests to prevent fatigue.

\section{Outcome measures}

\section{Community ambulation levels}

This method was presented by Rosa et al. [20] concerning the difficulties experienced in outdoor ambulation after stroke, which is a self-reporting questionnaire that allows the participants to describe their level of community ambulation ability. The questionnaire has 5 dimensions as follows [20]: (1) have no difficulty in walking in the community and 
do not require physical assistance or supervision; (2) mild difficulty in walking in the community, requiring supervision to walk far away from home; (3) moderate difficulty, needing supervision to walk near and far away from the home; (4) severe difficulty in walking in the community, always requiring physical assistance from another person; and (5) does not walk outside of the home. Subjects who responded to level 1 were divided up into "Independent Community Ambulation (ICA)". The others responding with level 2-5 were categorized as "Dependent Community Ambulation (DCA)".

\section{Timed up and go test}

The TUG test was used to examine the possibility for ICA, which reflects the functional mobility of subjects. The TUG can test one's movement and balance ability in an efficient manner. The TUG was performed as follows: participants sit on an armchair, and at the experimenter's start signal, they stand up from the chair, walk 3 meters ahead, return to the chair, and sit down, and their performance is timed by a physical therapist who was not involved in the subject's rehabilitation. The TUG test-retest reliability was reported as ICC $=0.96[1,3]$. During the test, physical assistance was not allowed except for use of an ambulation aid, such as a cane.

\section{Data analysis}

Data analysis was completed with the IBM SPSS Statistics ver. 18.0 (IBM Co., Armonk, NY, USA) program and the normality of variables was tested through the
Shapiro-Wilk method. Frequency analysis was done for the general characteristics of participants. Pearson's chi-squared test $\left(\chi^{2}\right)$ and independent $t$-tests were performed for the medical characteristics and functional performance results between groups.

For the discrimination of TUG between the ICA group and DCA group, the Youden index of receiver operating characteristic (ROC) curve was utilized to find out the optimal cut-off value. In the area under the ROC curve (AUC), the cases of $0.5<\mathrm{AUC} \leq 0.7$ were viewed as less accurate tests; $0.7<\mathrm{AUC} \leq 0.9$, fairly accurate tests; $0.9<\mathrm{AUC}<1.0$, very accurate tests; and $\mathrm{AUC}=1$, perfect tests [21]. Additionally, the Binomial Logistic Regression Model was utilized to produce the likelihood ratio of selected TUG cut-off values for the determination of CAL. The statistical significance level was set at $\alpha=0.05$. To examine for a suitable sample size for the study, the G*Power software ver. 3.0.10 (Franz Faul, Universitat Kiel, Germany) was used. Total sample size was estimated to be 118 patients based on coefficient of determination $\mathrm{r}^{2}=0.08, \alpha=0.05$, power $=0.80$, effect size $r=0.283$, and drop rate $25 \%$.

\section{Results}

The clinical significance of this study was finding that a TUG performance time of $<14.87$ seconds is a sufficient evaluation criterion for discrimination validity to recognize ICA abilities in persons with chronic stroke. In addition, the TUG test can serve as a factor in discriminating patients' ambulation velocity, falling, and community ambulation

Table 1. General characteristics

$(\mathrm{N}=87)$

\begin{tabular}{lccc}
\hline \multicolumn{1}{c}{ Characteristic } & ICA group $(\mathrm{n}=35)$ & DCA group $(\mathrm{n}=52)$ & $p$-value \\
\hline Sex (male/female) & $24 / 11$ & $25 / 27$ & $0.078^{\mathrm{a}}$ \\
Age (yr) & $54.09(13.81)$ & $58.52(9.88)$ & $0.107^{\mathrm{a}}$ \\
Disease period (mo) & $12.97(8.54)$ & $16.92(10.32)$ & $0.064^{\mathrm{a}}$ \\
Cause (cerebral infarction/hemorrhage) & $21 / 14$ & $41 / 11$ & $0.090^{\mathrm{a}}$ \\
Paralysis type (left/right) & $19 / 16$ & $28 / 24$ & $0.968^{\mathrm{a}}$ \\
Community ambulation level & & & $0.001^{\mathrm{a}}$ \\
1/2/3/4/5 & $35 / 0 / 0 / 0 / 0$ & $0 / 17 / 28 / 7 / 0$ & $0.002^{\mathrm{a}}$ \\
Use of aid & & & 14 \\
Independent ambulation & 23 & 31 & 7 \\
Cane & 10 & $33.05(21.67)$ & $0.001^{\mathrm{b}}$ \\
4-legged cane & $11.25(6.31)$ & & \\
Timed up and go (s) & & & \\
\hline
\end{tabular}

Values are presented as only number or mean (SD).

ICA: independent community ambulation, DCA: dependent community ambulation.

${ }^{\mathrm{a}}$ Pearson chi-square. ${ }^{\mathrm{b}}$ Independent t-tests. 
abilities.

Out of 120 patients, 102 had met the criteria and participated in the study. Seven of them had dropped out in the middle of the test, four had withdrawn due to health deterioration, and four were discharged from the hospital unexpectedly. The final number of subjects who had participated was 87 . The study showed no differences in sex, age, disease period, and paralysis pattern among the general medical characteristics between groups (Table 1). However, significant differences were found in their survey answers regarding the use of aids and community ambulation $(p<0.05$; Table 1). The TUG performance times of the ICA group and DCA group were 11.25 seconds and 33.05 seconds, respectively, showing a significant difference $(p<$ $0.05)$.

The optimal TUG cut-off value to differentiate the community ambulation abilities of subjects was $<14.87$ seconds (AUC $=0.871,95 \% \mathrm{CI}=0.797-0.945$ ) with mid-level accuracy. It was found that the method is sufficiently discriminative for recognizing the differences in diverse CAL.

In identifying the independence of community ambulation cases of those with chronic stroke, the diagnosis technique was found to have $80.0 \%$ susceptibility of selected TUG cut-off value, $78.8 \%$ singularity, and $71.8 \% / 85.4 \%$ positive/ negative prediction, respectively, producing excellent results (Table 2).

In the likelihood ratio of the selected TUG cut-off value for discriminating community ambulation independence, the Binomial Logistic Regression Model showed a significant difference $(p=0.006)$. The group with a TUG performance time of less than 14.87 seconds showed a 2.889 times higher probability of ICA than the group with a performance time greater than 14.87 seconds.

\section{Discussion}

When patients with stroke are discharged from the hospi- tal or returned to the community, it is essential for clinical practitioners to inform patients whether they can ambulate independently in the community. Therefore, the clinicians should identify relevant variables and assessment tools to predict independence of ambulation. Community gait ability is related to several factors such as perception, balance, and muscle strength [22]. The TUG test consists of consecutive tasks as standing up from a chair, walking forward, turning, coming back, and sitting down on the chair. TUG tasks demands one to interact with the surrounding environment, as well as have integrative upper/lower-limb movement with a coordinated cognitive process. In general, when patients perform the TUG test, they need to have the neuromuscular-skeletal components of agility, flexibility, balance, and strength $[12,18,19]$. This present study sought to investigate any relationship between the TUG performance time and CAL in chronic stroke survivors.

This study has found that the TUG cut-off value to recognize the differences in CAL had mid-level accuracy within acceptable range. Podsiadlo and Richardson [1] and Shumway-Cook et al. [23] reported the cut-off value for TUG performance time for healthy adults and people with a disease. Podsiadlo and Richardson [1] stated that if older people have a TUG performance time of $<20$ seconds, they could independently perform the necessary movements in activities of daily living; if it was $>30$ seconds, the person depended on an aid for ambulation in most activities. According to the criterion established by Podsiadlo and Richardson [1], 49 (56.3\%) out of 87 participants recorded a TUG performance time of $<20$ seconds, indicating that they were capable of independent performance and community participation. On the other hand, 24 (27.6\%) participants had a TUG performance time of $>30$ seconds, meaning that they were dependent on an aid for performing daily activities and experienced restrictions in ambulation. Bischoff $e t$ al. [24] investigated 413 older community female residents and 78 facility female residents who could move. They con-

Table 2. Susceptibility, singularity and positivity/negativity prediction for differentiating community ambulation levels $(\mathrm{N}=87)$

\begin{tabular}{|c|c|c|c|c|c|c|c|c|}
\hline \multicolumn{2}{|c|}{ Variable } & \multirow{2}{*}{$\begin{array}{c}\begin{array}{l}\text { ICA group } \\
(\mathrm{n}=35)\end{array} \\
28\end{array}$} & \multirow{2}{*}{$\begin{array}{c}\begin{array}{c}\text { DCA group } \\
(\mathrm{n}=52)\end{array} \\
11\end{array}$} & \multirow{2}{*}{$\frac{p \text {-value }}{0.001}$} & \multirow{2}{*}{$\begin{array}{l}\begin{array}{c}\text { Susceptibility } \\
(\mathrm{n}=35)\end{array} \\
28(80.0)\end{array}$} & \multirow{2}{*}{$\begin{array}{l}\begin{array}{c}\text { Singularity } \\
(\mathrm{n}=52)\end{array} \\
41(78.8)\end{array}$} & \multirow{2}{*}{$\begin{array}{c}\begin{array}{c}\text { Positive } \\
\text { prediction } \\
(\mathrm{n}=39)\end{array} \\
28(71.8)\end{array}$} & \multirow{2}{*}{$\begin{array}{c}\begin{array}{c}\text { Negative } \\
\text { prediction } \\
(\mathrm{n}=48)\end{array} \\
41(85.4)\end{array}$} \\
\hline TUG (sec) & $\begin{array}{l}<14.87 \\
\geq 14.87\end{array}$ & & & & & & & \\
\hline
\end{tabular}

Values are presented as number only or number (\%).

Chi-square test.

ICA: independent community ambulation, DCA: dependent community ambulation, TUG: timed up and go test. 
ducted the TUG test and stated that those with scores of $>12$ seconds on the initial test had a mobility problem, and therefore had needed a therapeutic intervention. Based on the TUG performance time cut-off value established by Bischoff et al. [24] (>12 seconds), 27 participants (31.0\%) of the 87 participants were capable of community ambulation, whereas the remaining 60 participants $(69.0 \%)$ were not. According to a survey on CAL in this study, the ICA group included 35 persons (40.2\%), and the DCA group included 52 persons (59.8\%). Their TUG performance times were 11.25 seconds and 33.05 seconds, respectively, representing a significant difference. By referring to the study findings of Podsiadlo and Richardson [1] and Bischoff et al. [24], the cut-off value of the TUG performance time was $<14.87$ seconds for this study in order to differentiate the participants' community ambulation ability levels.

TUG task includes diverse components different from ambulation velocity and distance. Similarly to this, in the real community, most of the activities are multi-tasked [25]. In terms of this, to perform the TUG test, lower limb muscle strength, balance, and attention span is needed [10]. Since physical efficiency is a basic factor contributing in community ambulation, our results were extent to previous literatures in basis on measurement of community ambulation.

We could not measure the qualitative aspects of asymmetrical body weight loading and posture agitation and psychological factors, such as the self-balance confidence, that could have affected the level of community ambulation. The category of community ambulation was dependent on the subject's response, and thus, it may be somewhat underestimated or overestimated. In our study, $45.9 \%$ of subjects used an ambulation aid during the TUG test, limiting the generalization of this study's findings. Nevertheless, the present study found that the TUG method is discriminative in classifying community ambulation of chronic stroke survivors. We recommend to physical therapists that the TUG can be utilized as a basic factor of community ambulation measurement in the clinical field. In further research, we would like to consider external factors, as social roles and life situations in measurement of community ambulation.

The TUG test consists of consecutive tasks and demands one's interaction with the surrounding environment and integrative upper/lower-limb movement with a coordinated cognitive process. The TUG cut-off value presented in this study may serve as the basis for future determination of ambulation levels necessary for community participation and should be available in clinical settings.

\section{Conflict of Interest}

The authors declared no potential conflicts of interest with respect to the authorship and/or publication of this article.

\section{References}

1. Podsiadlo D, Richardson S. The timed "Up \& Go": a test of basic functional mobility for frail elderly persons. J Am Geriatr Soc 1991;39:142-8.

2. Flansbjer UB, Holmbäck AM, Downham D, Patten C, Lexell J. Reliability of gait performance tests in men and women with hemiparesis after stroke. J Rehabil Med 2005;37:75-82.

3. Norén AM, Bogren U, Bolin J, Stenström C. Balance assessment in patients with peripheral arthritis: applicability and reliability of some clinical assessments. Physiother Res Int 2001;6:193204.

4. Berg KO, Maki BE, Williams JI, Holliday PJ, Wood-Dauphinee SL. Clinical and laboratory measures of postural balance in an elderly population. Arch Phys Med Rehabil 1992;73:1073-80.

5. Persson CU, Danielsson A, Sunnerhagen KS, Grimby-Ekman A, Hansson PO. Timed Up \& Go as a measure for longitudinal change in mobility after stroke - Postural Stroke Study in Gothenburg (POSTGOT). J Neuroeng Rehabil 2014;11:83.

6. van Iersel MB, Munneke M, Esselink RA, Benraad CE, Olde Rikkert MG. Gait velocity and the Timed-Up-and-Go test were sensitive to changes in mobility in frail elderly patients. J Clin Epidemiol 2008;61:186-91.

7. Yan T, Hui-Chan CW. Transcutaneous electrical stimulation on acupuncture points improves muscle function in subjects after acute stroke: a randomized controlled trial. J Rehabil Med 2009; 41:312-6.

8. Ng SS, Hui-Chan CW. Does the use of TENS increase the effectiveness of exercise for improving walking after stroke? A randomized controlled clinical trial. Clin Rehabil 2009;23:1093103.

9. Persson CU, Hansson PO, Sunnerhagen KS. Clinical tests performed in acute stroke identify the risk of falling during the first year: postural stroke study in Gothenburg (POSTGOT). J Rehabil Med 2011;43:348-53.

10. Shumway-Cook A, Brauer S, Woollacott M. Predicting the probability for falls in community-dwelling older adults using the Timed Up \& Go Test. Phys Ther 2000;80:896-903.

11. O'Brien K, Culham E, Pickles B. Balance and skeletal alignment in a group of elderly female fallers and nonfallers. J Gerontol A Biol Sci Med Sci 1997;52:B221-6.

12. Ng SS, Hui-Chan CW. The timed up \& go test: its reliability and association with lower-limb impairments and locomotor capacities in people with chronic stroke. Arch Phys Med Rehabil 2005;86:1641-7.

13. Faria CD, Teixeira-Salmela LF, Nadeau S. Effects of the direction of turning on the timed up \& go test with stroke subjects. Top Stroke Rehabil 2009; 16:196-206.

14. Lord SE, McPherson K, McNaughton HK, Rochester L, Weatherall M. Community ambulation after stroke: how im- 
portant and obtainable is it and what measures appear predictive? Arch Phys Med Rehabil 2004;85:234-9.

15. World Health Organization. International classification of functioning, disability and health (ICF). Geneva: World Health Organization; 2001.

16. Patla AE, Shumway-Cook A. Dimensions of mobility: defining the complexity and difficulty associated with community mobility. J Aging Phys Act 1999;7:7-19.

17. Shumway-Cook A, Patla AE, Stewart A, Ferrucci L, Ciol MA, Guralnik JM. Environmental demands associated with community mobility in older adults with and without mobility disabilities. Phys Ther 2002;82:670-81.

18. Miotto JM, Chodzko-Zajko WJ, Reich JL, Supler MM. Reliability and validity of the fullerton functional fitness test: an independent replication study. J Aging Phys act 1999;7:339-53.

19. Schenkman M, Hughes MA, Samsa G, Studenski S. The relative importance of strength and balance in chair rise by functionally impaired older individuals. J Am Geriatr Soc 1996;44:1441-6.

20. Rosa MC, Marques A, Demain S, Metcalf CD. Fast gait speed and self-perceived balance as valid predictors and discriminators of independent community walking at 6 months post-stroke--a preliminary study. Disabil Rehabil 2015;37:129-34.

21. Granger CV, Albrecht GL, Hamilton BB. Outcome of comprehensive medical rehabilitation: measurement by PULSES profile and the Barthel Index. Arch Phys Med Rehabil 1979;60: $145-54$.

22. Lee KB, Lim SH, Ko EH, Kim YS, Lee KS, Hwang BY. Factors related to community ambulation in patients with chronic stroke. Top Stroke Rehabil 2015;22:63-71.

23. Shumway-Cook A, Baldwin M, Polissar NL, Gruber W. Predicting the probability for falls in community-dwelling older adults. Phys Ther 1997;77:812-9.

24. Bischoff HA, Stähelin HB, Monsch AU, Iversen MD, Weyh A, von Dechend $\mathrm{M}$, et al. Identifying a cut-off point for normal mobility: a comparison of the timed 'up and go' test in community-dwelling and institutionalised elderly women. Age Ageing 2003;32:315-20.

25. Hwang WJ, Cho MK, Chung Y. Relationship between anticipatory postural adjustment of the trunk, dual tasks and physical performance with chronic stroke survivors: a pilot test. Phys Ther Rehabil Sci 2015;4:44-8. 